\title{
The intrinsic equatorial ellipticity of bulges
}

\author{
J. Méndez-Abreu ${ }^{1}$, J. A. L. Aguerri ${ }^{2}$, E. M. Corsini ${ }^{3}$ and \\ E. Simonneau ${ }^{4}$ \\ ${ }^{1}$ INAF-Osservatorio Astronomico di Padova, vicolo dell'Osservatorio 5, I-35122 Padova, Italy \\ email: jairo.mendez@oapd.inaf.it \\ ${ }^{2}$ Instituto de Astrofísica de Canarias, C/ Vía Láctea s/n, E-38200 La Laguna, Spain \\ email: jalfonso@iac.es \\ ${ }^{3}$ Dipartimento di Astronomia, Università di Padova, vicolo dell'Osservatorio 3, I-35122 \\ Padova, Italy \\ email: enricomaria.corsini@unipd.it \\ ${ }^{4}$ Institute d'Astrophysique de Paris, C.N.R.S., 98 bis Boul. Arago, F-75014 Paris, France
}

\begin{abstract}
Traditionally, the study of the structural parameters of galaxies have been used to understand the bulge formation processes. However, one piece lost in this study is the threedimensional shape of the bulges. In this work, the structural parameters of a magnitude-limited sample of 148 unbarred S0-Sb galaxies were derived to study the correlations between bulge and disk parameters as well as the probability distribution function (PDF) of the intrinsic equatorial ellipticity of bulges. A new algorithm (GASP-2D) was used to perform the bidimensional bulge-disk decomposition of $J$-band galaxy images extracted from the archive of the 2MASS survey. The PDF of intrinsic ellipticities was derived from the distribution of the observed ellipticities of bulges and misalignments between bulges and disks. About $80 \%$ of bulges in $\mathrm{S} 0-\mathrm{Sb}$ galaxies are not oblate but triaxial ellipsoids. Their mean axial ratio in the equatorial plane is $\langle B / A\rangle=0.85$. There is not significant dependence of their PDF on morphology, light concentration, and luminosity. The scenarios in which bulges assembled from mergers and/or grew over long times through disk secular evolution have to be tested against the derived PDF of bulge intrinsic ellipticities.
\end{abstract}

Keywords. galaxies: bulges, galaxies: evolution, galaxies: formation, galaxies: fundamental parameters, galaxies: photometry, galaxies: statistics, galaxies: structure

\section{Correlations between the structural parameters of bulges and disks}

The bulge and disk structural parameters of a magnitude limited sample of 148 unbarred S0-Sb galaxies were derived using a new fitting code (GASP-2D, Méndez-Abreu et al. 2007). A series of correlations were found for both bulge and disk parameters. Larger bulges have surface brightness radial profiles which are more centrally peaked than that of the smaller bulges, moreover they have a lower effective surface brightness and higher absolute luminosities. Larger disks have a lower central surface brightness. Furthermore, larger disks reside in large bulges revealing a strong coupling between bulges and disks.

Central velocity dispersions are available for a subsample of galaxies. Their bulges are consistent with the Fundamental (Djorgovski \& Davis 1987) and Photometric (Khosroshahi et al. 2000) Planes of bright elliptical galaxies.

Finally, we found a new correlation between the disk scale length and the central velocity dispersion of galaxies, implying that larger disks are located in galaxies with most massive bulges. 

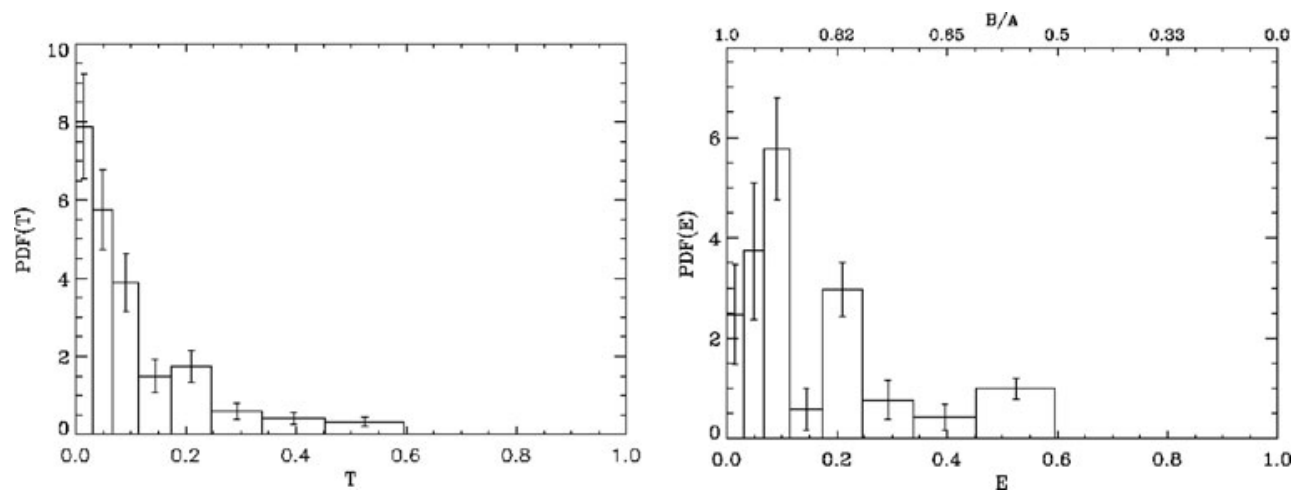

Figure 1. Left panel: PDF of $T . T$ is our observable and was calculated using the twist angle $(\delta)$ between bulge and disk, bulge ellipticity $(\epsilon)$ and galaxy inclination $(\theta)$. Right panel: PDF of $E$ obtained from the inversion of $T$. $E$ is related to the intrinsic axis ratio by $E=(1-B / A) /(1+B / A)$ where $A$ and $B$ are the semi-axis of the bulge ellipsoid in the equatorial plane. In both panels the probability is normalized over 10 bins geometrically distributed to cover the interval $[0,1]$. The width of the first bin is 0.03 and the width ratio of two consecutive bins is 1.25 . Error bars correspond to a Poissonian statistics.

\section{The equatorial intrinsic ellipticity of bulges}

The determination of the intrinsic shape of bulges is an ill-posed problem, that can be tackled only in a statistical way. A new approach, that allow us to derive the probability distribution function of intrinsic ellipticities $\operatorname{PDF}(E)$ using only photometric data was used (see Méndez-Abreu et al. 2007, for further details). Fig 1. show the PDF obtained from our galaxy sample, the significant decrease of probability for $E<0.07$ (or equivalently $B / A<0.93)$ suggests that the shape of bulge ellipsoids in their equatorial plane is most probably elliptical rather than circular. A series of test confirms that such a decrease is due to neither by the lack of statistics nor by the width of the bins. The average ellipticity $\langle E\rangle$ weighted with the PDF was calculated obtaining a value of $\langle E\rangle=0.16$ (〈 $B / A\rangle=0.85)$. This is consistent with previous findings by Bertola et al. (1991) and Fathi \& Peletier (2003). The $\operatorname{PDF}(E)$ shows that there are not bulges with $E>0.6(B / A<0.5)$ and it is not related to morphology, light concentration, or luminosity of bulges.

\section{Discussion and conclusions}

According to their structural parameters, the bulges of our sample galaxies follow the same Fundamental and Photometric Plane of elliptical galaxies, supporting the idea that bulges and ellipticals are formed in the same way. On the other hand, the correlations between bulge and disk parameters, are usually interpreted as an indication of secular evolution processes. These results indicate that the above relations are not enough to distinguish between bulges formed by early dissipative collapse, merging or secular evolution. All these mechanisms could be tested against the PDF of the intrinsic equatorial ellipticity of bulges.

\section{References}

Bertola, F., Vietri, M., \& Zeilinger, W. W. 1991, ApJ (Letters), 374, L13

Djorgovski, S. \& Davis, M. 1987, ApJ, 313, 59

Fathi, K. \& Peletier, R. F. 2003, A\&A, 407, 61

Khosroshahi, H. G., Wadadekar, Y., \& Kembhavi, A. 2000, ApJ, 533, 162

Méndez-Abreu, J., Aguerri, J. A. L., Corsini, E. M. \& Simonneau, E. 2007, A\&̈A (submitted) 